\title{
Alargamiento Oseo Con Clavo Intramedular Magnetico Experiencia Preliminar y Lecciones Aprendidas
}

\section{Bone Lengthening with Magnetic Intramedullary Nail Preliminary Experience and Learned Lessons}

\author{
Alejandro Baar-Zimend ${ }^{1}$ \\ 1 Jefe de la Unidad Ortopedia Y Traumatologia Infantil Clinica Las \\ Condes, Santiago, Region Metropolitana, Chile \\ Rev Chil Ortop Traumatol 2020;61:60-68.
}

\author{
Address for correspondence Alejandro Baar-Zimend, MD, \\ Departamento de Ortopedia Y Traumatología, Clínica Las Condes, Lo \\ Fontecilla 441 Las Condes CP 7591046, Chile \\ (e-mail: abaarz@gmail.com).
}

\section{Resumen \\ Palabras clave \\ - alargamiento óseo \\ - osteogénesis por distracción \\ - clavo intramedular magnético}

Objetivos Mostrar resultados, complicaciones y lecciones aprendidas utilizando el clavo intramedular magnético (IML, Intramedullary Magnetic Lengthener) en un grupo de pacientes sometidos a alargamiento óseo.

Pacientes y Métodos Entre enero 2017 y diciembre 2019, 9 pacientes (15 segmentos), edad entre 15 y 39 años, fueron sometidos a alargamiento óseo con IML: 5 pacientes tuvieron alargamiento femoral bilateral por talla baja, 1 paciente se sometió a alargamiento de Piernas bilateral por Tibia vara y acortamiento mesomélico y 3 recibieron alargamientos femorales unilaterales por discrepancia de longitud de extremidades. Todos fueron operados por el mismo cirujano, con técnica standard. Se indicó kinesiterapia al menos 5 veces por semana durante la fase de distracción.

Resultados En todos se logró el objetivo de alargamiento planteado. No hubo complicaciones intra ni postoperatorias graves (TVP, TEP, Embolia grasa), ni fallas del sistema distractor. Un paciente desarrolló contracturas articulares de ambas rodillas por no adhesión a Kinesiterapia. Ninguno requirió aporte de injerto óseo, sin embargo en 2 pacientes de alargamiento de Fémur bilateral, se presentó deformidad en varo, que hizo necesario recambio a clavo convencional y un paciente desarrolló una parálisis transitoria del Nervio Peroneo común.

Conclusiones El advenimiento de los IML significó un gran avance en el campo de la osteogènesis por distracción, sin embargo, aún se trata de un procedimiento complejo, que debe ser planificado cuidadosamente para minimizar los riesgos y complicaciones. Una selección meticulosa del paciente y la evaluación física y psicológica previa, son fundamentales para el éxito del procedimiento. Los casos bilaterales requieren de un seguimiento cercano, por el riesgo de falla del implante.

\section{Abstract}

Objective We show our results, complications and lessons learned using the Intramedullary Magnetic Lengthener (IML) in a group of patients who were subjected to bone lengthening.

Patients and Methods Between January 2017 and December 2019, 9 patients (15 segments), aged between 15 and 39 years, underwent bone lengthening with IML: 5

received

April 13, 2020

accepted

June 19, 2020
DOI https://doi.org/

10.1055/s-0040-1715160. ISSN $0716-4548$.
Copyright $\odot 2020$ by Thieme Revinter

Publicações Ltda, Rio de Janeiro, Brazil
License terms

c) $9 \ominus \$$ 


\author{
Keywords \\ - bone lengthening \\ - distraction \\ osteogenesis \\ - magnetic \\ intramedullary \\ nail
}

patients had bilateral femoral lengthening due to short stature, 1 patient had bilateral leg lengthening due to Tibia vara and mesomelic shortening and 3 had unilateral femoral lengthening for limb length discrepancy. All were operated by the same surgeon, with standard technique. Physical therapy was indicated at least 5 times per week during the distraction phase.

Results In every case, the proposed lengthening amount was achieved. There were no serious intraoperative or postoperative complications (DVT, Pulmonary Embolism, Fat Embolism), nor failures of the distractor system. One patient developed knee joint contracture due to non-adherence to Physical therapy. None required bone grafting, however, in 2 patients with bilateral Femur, a varus deformity appeared, which required nail exchange with a conventional trauma nail. One case developed a transit common Peroneal Nerve palsy.

Conclusions IMLs represent a great advance in the bone lengthening realm, however, it is still a complex procedure, which must be carefully planned to minimize risks and complications. Meticulous patient selection and prior physical and psychological evaluation are essential to the success of the procedure. Bilateral cases need a closer follow up, because implant failure is a potential risk.

\section{Introducción}

El alargamiento óseo mediante osteogénesis por distracción (OD) es una técnica ya establecida dentro del campo de la cirugía ortopédica. Aunque existen reportes de casos a partir del año $1905,{ }^{1}$ no fue si no hasta fines de la década de los 50s, que alcanzó su apogeo, gracias a los trabajos de Ilizarov, en Kurgan, ex URSS. ${ }^{2}$

Utilizando componentes simples, Ilizarov creó un aparato que revolucionó la cirugía ortopédica para siempre: el fijador externo circular. Su versatilidad, ha permitido desde entonces corregir deformidades que parecían imposibles, curar infecciones inveteradas y alargar segmentos óseos en las más diversas condiciones: congénitas, post traumática, e incluso cosméticas. A pesar de sus enormes ventajas, su uso requiere una curva de aprendizaje importante, y los tratamientos no están exentos de complicaciones, que dependiendo de las series oscilan entre 25 y $75 \%{ }^{3-5}$ e incluyen: Infección de pins, dolor, rigidez articular, incomodidad, falla de materiales, entre otras.

Para evitar los problemas descritos, ya desde principios de los 80s hubo intentos por desarrollar clavos intramedulares con la capacidad de realizar OD, sin necesidad de elementos de transifixión ósea. ${ }^{6,7}$ El primero de esos dispositivos se adjudica a Bliskunov, en Ucrania. Posteriormente el clavo Albizzia, diseñado por Guichet en Francia y el ISKD (Orthofix) diseñado por Cole, en USA. Los clavos antes mencionados, corresponden a la primera generación, con un mecanismo de trinquete, que requería manipulación externa de la extremidad para lograr distracción ósea. En 1991, nace el primer clavo motorizado, diseñado por Baumgart y Betz, en Alemania. ${ }^{8} \mathrm{~A}$ diferencia de los anteriores, por primera vez se introdujo un motor al implante, evitando la necesidad de manipular la extremidad manualmente, para lograr alargamiento.

En 2011 aparece el primer clavo de la tercera generación, Precice ${ }^{\circledR}$ (NuVasive Specialized Orthopedics, Inc., California,
USA) cuyo mecanismo de acción es a través de un campo magnético. La gran ventaja respecto a sus predecesores, es su capacidad de permitir tanto distracción como compresión. A partir de entonces, se han hecho mejoras considerables en el implante, aumentando su resistencia mecánica, y disminuyendo la tasa de complicaciones.

Es importante destacar que todos esos implantes intrtamedulares antes mencionados, son rectos, y no siguen la curvatura anterior fisiológica del Fémur, como ocurre con los clavos convencionales de trauma. Es por ello, que como parte de la planificación preoperatoria, debe decidirse entre un clavo corto (aquel que alcanza el nivel del apex del antecurvatum fisiológico) y uno largo (que permite abarcar el Fémur hasta la metáfisis distal). Para poder utilizar ese último, es imperativo que la osteotomía se realice a nivel del apex de la curvatura del Fémur.

En el presente trabajo, queremos mostrar nuestra experiencia preliminar en una serie de 9 pacientes sometidos a alargamiento óseo con clavo Precice, comentar las complicaciones y compartir las lecciones aprendidas.

\section{Pacientes y Métodos}

Entre enero 2017 y diciembre 2019, 9 pacientes, entre 15 y 38 años de edad, 6 de sexo masculino y 3 de sexo femenino, fueron sometidos a una osteotomía para DO con clavo Precice en 15 segmentos óseos. En 5 pacientes se realizó alargamiento bilateral de Fémur. Cuatro de ellos eran hombres y 1 mujer. Esa última, además presentaba torsion femoral interna severa. En 3 pacientes, portadores de discrepancia de longitud de extremidades, se realizó alargamiento Femoral único: 1 hombre y 2 mujeres. Una de ellas presentaba una deformidad en valgo, por lo que se utilizó clavo retrógrado. Finalmente, 1 paciente con acortamiento mesomélico de las Tibias asociado a 
Tabla 1 Características demográficas y clínicas de los pacientes

\begin{tabular}{|l|l|l|l|l|l|}
\hline $\mathbf{N}^{\circ}$ Caso & Edad & Género & Diagnostico & Procedimiento & Tipo clavo \\
\hline 1 & 18 & M & Talla Baja $(165 \mathrm{~cm})$ & Fémur bilateral & FAP \\
\hline 2 & 15 & F & Talla Baja $(154 \mathrm{~cm})+$ antetorsión severa & Fémur bilateral & FAT \\
\hline 3 & 38 & M & Talla Baja $(163 \mathrm{~cm})$ & Fémur bilateral & FAP \\
\hline 4 & 20 & M & Talla Baja $(162 \mathrm{~cm})$ & Fémur bilateral & FAP \\
\hline 5 & 16 & M & Talla Baja $(162 \mathrm{~cm})$ & Fémur bilateral & FAT \\
\hline 6 & 15 & F & DL por Encondromatosis & Fémur unilateral & FAT \\
\hline 7 & 20 & M & DL idiopático & Tibia Bilateral & TAP \\
\hline 8 & 18 & M & $\begin{array}{l}\text { Tibia Vara } \\
\text { Acortamiento Mesomélico }\end{array}$ & Fémur unilateral & FR \\
\hline 9 & 35 & F & $\begin{array}{l}\text { DL secuela lesión fisiaria } \\
\text { Valgo Fémur distal }\end{array}$ \\
\hline
\end{tabular}

Abreviaturas: DL, discrepancia longitud; F: femenino; FAP, Fémur anterógrado entrada piriforme; FAT, Fémur anterógrado entrada trocantérica; FR: Fémur retrógrado; M, masculino; T: clavo de Tibia.

deformidad en varo, fue sometido a alargamiento de ambas Piernas.

Para el caso de los alargamientos bilaterales de Fémur, el objetivo planteado fueron $8 \mathrm{~cm}$. Para el caso de Piernas bilateral, solo se recomendó $6,5 \mathrm{~cm}$. El resto, fue alargado de acuerdo a la discrepancia de longitud (-Tabla 1).

Previo a la operación, a todos los pacientes se les solicitaron exámenes generales (hemograma, perfil bioquímico, niveles plasmáticos de vitamina $\mathrm{D}$ y pruebas de coagulación) y teleradiografía (TeleRX) de extremidades inferiores (EEII), en proyección AP y LAT. Para aquellos que fueron sometidos a alargamiento bilateral, además se solicitó una evaluación por la psicóloga de nuestra unidad, de manera de pesquisar condiciones en las cuales estaría contraindicada esa operación (Dismorfofobia, Trastorno Bipolar, Esquizofrenia y algunos trastornos de personalidad). Todas las cirugías fueron realizadas por el autor. En los casos bilaterales, en mayores de 18 años, se realizó osteotomía a nivel subtrocantérico y se utilizó clavo corto, para evitar el paso por la curvatura anterior fisiológica del fémur. En menores de 18 años, debido a su mejor potencial de regeneración, se realizó la osteotomía en zona de apex de curvatura anterior del Fémur, lo que permitió utilizar clavos largos. En todos los casos, se utilizó profilaxis antibiótica con Cefazolina por 24 horas y antitrombótica por 15 días con Rivaroxaban $10 \mathrm{mg}$, y se instruyó realizar kinesiterapia motora diaria (al menos 5 veces por semana) durante la fase de distracción. Durante todo el tratamiento, y hasta la consolidación, los pacientes recibieron suplemento de Vitamina D 800 UI más Calcio 1200 mg al día.

Cuando se planificó el alargamiento femoral mayor a $5 \mathrm{~cm}$, se realizó adicionalmente sección del tracto iliotibial a nivel del polo distal de Patela. En el alargamiento bilateral de Tibias, se realizó fasciotomia percutánea del compartimento anterior profiláctica.

Para las Osteotomías de Fémur, la DO se comenzó a la semana post operatoria en mayores de 20 años y al día siguiente en menores de 20 años. Para el caso de las Tibias, la DO se postergó por 14 días desde el post operatorio.
Todos los fémures se alargaron a razón de 0,33 $\mathrm{mm} 3$ veces al día. En el caso de las Tibias, se utilizó un ritmo de $0.20 \mathrm{~mm}$ 3 veces al día.

Durante el primer mes, se controló semanalmente a los pacientes, y luego cada 2 semanas con radiografías, hasta alcanzar el objetivo planteado. Luego de eso, el control radiográfico fue mensual hasta la consolidación completa (-Figura 1).

Durante la DO, los pacientes fueron instruidos respecto a la carga de peso: para los casos bilaterales, se fue mucho más estricto, permitiendo apoyo parcial de acuerdo al diámetro del clavo utilizado (Para clavo 10,7 mm máximo $20 \mathrm{Kg}$ por pierna, para el de $12,5 \mathrm{~mm}$ máximo $30 \mathrm{Kg}$ por pierna) pero con apoyo ya sea en andador o con bastones.

En el caso de las piernas, la carga autorizada fue incluso menor, permitiendo máximo $15 \mathrm{Kg}$ por extremidad.

La presencia de al menos 3 corticales, en 2 proyecciones radiográficas ortogonales fue criterio para considerar consolidación y permitir la carga sin bastones.

\section{Resultados}

En todos los pacientes se logró completar satisfactoriamente el alargamiento planificado y no se produjeron fallas del mecanismo distractor durante la evolución.

En promedio, el alargamiento fue de $7,25 \mathrm{~cm}$ (Rango $2,5-8 \mathrm{~cm})$

El tiempo quirúrgico promedio fue de 130 minutos por segmento (rango 120-180 minute). El sangramiento intraoperatorio fue $183,33 \mathrm{cc}$ (rango 100-500 cc). En 2 de los pacientes, en que se realizó alargamiento unilateral de Fémur, fue necesario realizar osteotomía a cielo abierto: En un caso porque, simultáneamente, se retiró una placa de osteosíntesis (sangramiento $500 \mathrm{cc}$ ) (-Figura 2) y en el otro porque se realizó una corrección aguda de deformidad en valgo del Fémur distal (300 cc). Si excluimos esos casos, el sangramiento promedio fue de 121,45 cc. 


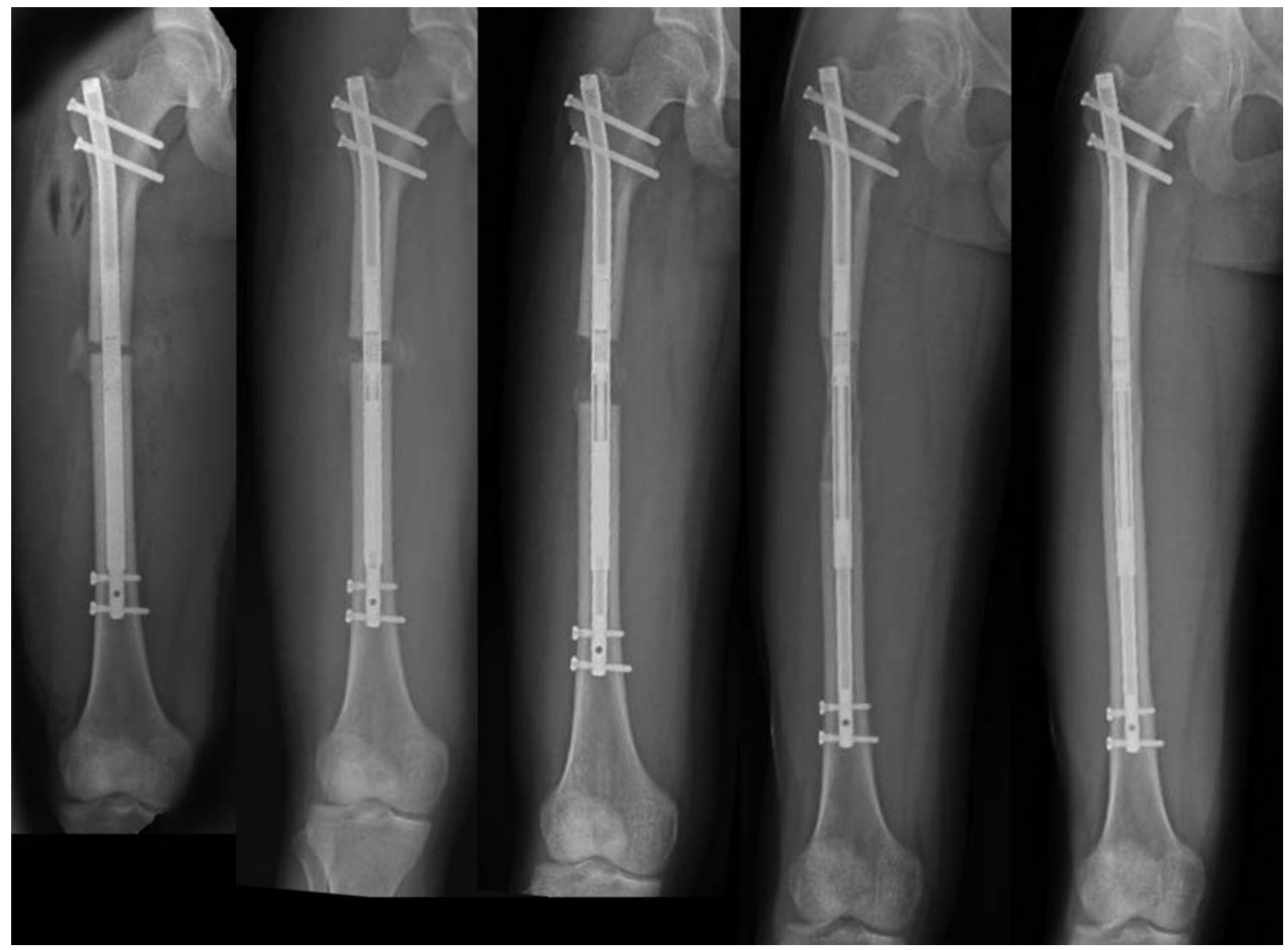

Fig. 1 Proceso de alargamiento de $80 \mathrm{~mm}$ con clavo Precice ${ }^{\circledR}$ hasta la consolidación completa.

De manera global, el índice de consolidación (HI, por su sigla Healing Index) fue de 46,5 días/cm (rango 23,1 - 98,7). Un hallazgo interesante es que en 4 de los 5 casos de alargamiento de Fémur, uno de los lados requirió más tiempo para consolidar que el contralateral. Los índices de consolidación de esos pacientes, para Fémur derecho versus izquierdo fueron: Paciente 1: 52,5 versus 36,6; Paciente 2: 28,5 versus 52,1 Paciente 3: 89,1 versus 98,7; Paciente 5: 23,1 versus 50,2. El Paciente 4 presentó un indice de consolidación de 26,2 días / $\mathrm{cm}$ en ambos Fémures. (-Tabla 2). En todos los casos que evolucionaron con una consolidación más lenta, se recomendó realizar maniobra de acordeón: Retroceder $0,5 \mathrm{~mm}$ en la mañana, Distracción $0,25 \mathrm{~mm}$ en la tarde y en la noche.

De acuerdo a la clasificación de Paley, ${ }^{3} 3$ pacientes se presentaron obstáculos y sólo hubo 1 complicación.

En 2 pacientes, con alargamiento bilateral de Fémur, fue necesario realizar recambio de uno de los clavos, por presentar retardo de consolidación asociado a deformación de varo del implante. En uno de ellos, el clavo presentaba fisuras en su componente proximal (-Figura 3 ).

Un paciente con alargamiento bilateral de Fémur, no siguió las recomendaciones de kinesiterapia ni asistió a los controles solicitados. A su tercera visita post operatoria, habiendo alcanzado los $8 \mathrm{~cm}$ de alargamiento, presentaba contractura en flexión de ambas caderas y rodillas. Ante tal escenario, se hospitalizó inmediatamente, se solicitó evaluación por
Fisiatría, y se realizó infiltración con Toxina botulínica en los músculos involucrados. Se indicó kinesiterapia intensiva intrahospitalaria, y luego en forma ambulatoria, sin embargo, el paciente no regresó a nuevos controles.

Finalmente, en el paciente sometido a alargamiento de ambas Tibias, durante la colocación de 1 de los tornillo de bloqueo proximal, se produjo estimulación del nervio Peroneo común. En el post operatorio inmediato, no hubo signos de lesión nerviosa, sin embargo, al tercer día post operatorio, comenzó con paresia y luego parálisis de extensores de tobillo y Peroneos. Se realizaron 2 ecografías, comprobando indemnidad en el trayecto del Nervio. Dado el carácter evolutivo, se decidió realizar descompresión del Nervio Peroneo.

El paciente evolucionó con recuperación motora y sensitiva luego de 12 meses, tiempo en el cual se indicó Ortesis de Tobillo/Pie.

\section{Discusión}

El desarrollo de dispositivos de alargamiento intramedular ha significado un cambio de paradigma en la cirugía de alargamiento óseo. Desde los primeros modelos hasta la nueva generación de implantes, los avances tecnológicos han permitido fabricar clavos de mayor resistencia, con mecanismos de distracción más fiables y controlados, sistemas de monitorización externa precisos, y con una muy 


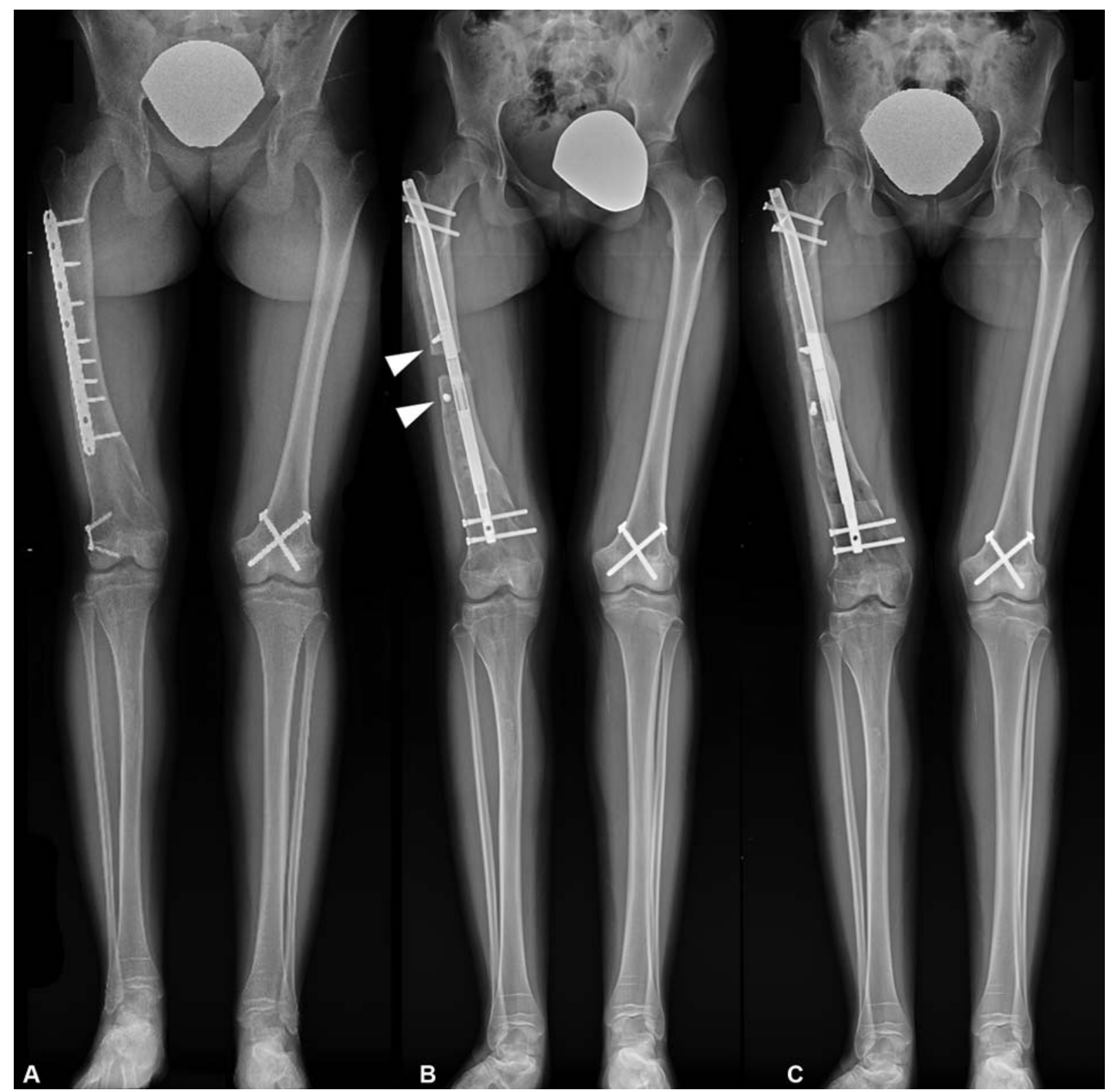

Fig. 2 Alargamiento de Fémur en Encondromatosis. (A): Discrepancia de longitud de Fémures. La placa se colocó después de sufrir una fractura. (B): Durante el alargamiento. Las puntas de flecha muestran los tornillos de bloqueo. (C): Resultado final.

baja tasa de fallas. ${ }^{9}$ Eso ha llevado a una tendencia cada vez mayor al uso de esos sistemas en desmedro de los fijadores externos. Aunque esos últimos tendrían la ventaja de permitir la corrección de deformidades en forma simultánea al alargamiento, existen varios trabajos que han demostrado la eficacia del uso de esos implantes para fijar correcciones agudas y luego realizar alargamiento progresivo. ${ }^{10-14}$ En esta serie, 3 pacientes (5 segmentos) presentaban deformidades concomitantes, que fueron corregidas durante la cirugía. La clave del éxito en esos casos, es el uso de tornillos de bloqueo (Poller screws). La planificación pre operatoria es fundamental en esos casos. ${ }^{15}$

Otra ventaja teórica de tutores externos por sobre los IML, es la posibilidad de utilizarlos en niños, por el riesgo de daño fisiario asociado a implantes intramedulares.

En un estudio realizado por Hammouda ${ }^{16}$ con 31 Fémures operados con clavo Precice de entrada trocantérica, en adolescentes de edad promedio 12,9 años, no se observaron casos de necrosis avascular (AVN) ni deformidad del fémur luego de 3,5 años de seguimiento. Frommer, ${ }^{17}$ en una serie con 60 clavos intramedulares instalados en adolescentes de 13,6 años promedio, no observó AVN ni deformidad a 10 meses de seguimiento. Gordon, ${ }^{18}$ en una serie de 37 procedimientos de alargamiento sobre clavo (LON) en pacientes de 11,6 años promedio, con seguimiento de 2 años, no observó AVN ni deformidades. Ese mismo autor, ${ }^{19}$ en 2003 concluyó que el enclavijado femoral transtrocantérico es un procedimiento seguro en niños mayores de 9 años.

Una limitación importante para el uso masivo de los IML es su valor comercial. Actualmente, su precio oscila entre los USD20.000 - 22.000, lo que hace muy difícil abandonar del todo los fijadores externos. Sin embargo, un estudio realizado por Richardson y col., ${ }^{20}$ comparando 
Tabla 2 Resultados clínicos del alargamiento

\begin{tabular}{|c|c|c|c|c|c|c|}
\hline $\mathrm{N}^{\circ}$ Caso & Diagnóstico & $\begin{array}{l}\text { Objetivo } \\
\text { Alargam. } \\
(\mathrm{mm})\end{array}$ & $\begin{array}{l}\text { Alargam. } \\
\text { logrado } \\
(\mathrm{mm})\end{array}$ & $\begin{array}{l}\text { Indice } \\
\text { Consolidación } \\
\text { (días/cm) }\end{array}$ & Complicaciones & $\begin{array}{l}\text { Manejo de la } \\
\text { complicación }\end{array}$ \\
\hline 1 & Talla Baja $(165 \mathrm{~cm})$ & 80 & 80 & $\begin{array}{l}\text { FD: } 52,5 \\
\text { Fl: } 36,3\end{array}$ & - & \\
\hline 2 & $\begin{array}{l}\text { Talla baja }(154 \mathrm{~cm})+ \\
\text { antetorsión severa }\end{array}$ & 80 & 80 & $\begin{array}{l}\text { FD: } 28,5 \\
\text { FI: } 52,1\end{array}$ & - & \\
\hline 3 & Talla Baja $(163 \mathrm{~cm})$ & 80 & 80 & $\begin{array}{l}\text { FD: } 89,1 \\
\text { FI: } 98,7\end{array}$ & $\begin{array}{l}\text { Deformidad en } \\
\text { Varo por } \\
\text { retardo consolidación. } \\
\text { Fatiga de implante } \\
\text { izquierdo }\end{array}$ & $\begin{array}{l}\text { Recambio a } \\
\text { clavo estándar }\end{array}$ \\
\hline 4 & Talla Baja (162 cm) & 80 & 80 & $\begin{array}{l}\text { FD: } 26,2 \\
\text { FI: } 26,2\end{array}$ & $\begin{array}{l}\text { Contractura en flexion } \\
\text { de caderas y rodillas }\end{array}$ & $\begin{array}{l}\text { Infiltración con } \\
\text { Toxina Botulínica }\end{array}$ \\
\hline 5 & Talla Baja $(162 \mathrm{~cm})$ & 80 & 80 & $\begin{array}{l}\text { FD: } 23,1 \\
\text { FI: } 50,2\end{array}$ & $\begin{array}{l}\text { Deformidad en Varo } \\
\text { por retardo de } \\
\text { consolidación. } \\
\text { Deformación del } \\
\text { implante izquierdo }\end{array}$ & $\begin{array}{l}\text { Recambio a } \\
\text { clavo estándar }\end{array}$ \\
\hline 6 & $\begin{array}{l}\text { DL por } \\
\text { Encondroma-tosis }\end{array}$ & 30 & 30 & FD: 26,7 & - & \\
\hline 7 & DL idiopático & 25 & 25 & FI: 38,4 & - & \\
\hline 8 & $\begin{array}{l}\text { Tibia Vara } \\
\text { Acortamiento } \\
\text { Mesomélico }\end{array}$ & 65 & 65 & $\begin{array}{l}\text { TD: } 51,2 \\
\text { TI: } 51,2\end{array}$ & Paresia N. Peroneo & $\begin{array}{l}\text { Descompresión N. } \\
\text { Peroneo y uso de } \\
\text { OTP hasta recuperación }\end{array}$ \\
\hline 9 & $\begin{array}{l}\mathrm{DL}+\text { Valgo } \\
\text { Fémur distal }\end{array}$ & 40 & 40 & $\begin{array}{l}3 \text { meses post op } \\
\text { En proceso } \\
\text { consolidación }\end{array}$ & - & \\
\hline
\end{tabular}

Abreviaturas: FD, Fémur derecho; FI, Fémur izquierdo; TD, Tibia derecha; TI, Tibia izquierda.

alargamientos realizados con IML versus LON, demostró que, a pesar del costo más elevado del IML, el valor total del procedimiento, considerando las complicaciones, la necesidad de reingreso a pabellón y los honorarios del cirujano era similar (USD 53.000 para IMP versus 48.000 para LON).

En nuestra serie, el tiempo operatorio fue de aproximadamente 120 minutos por cada segmento operado, y el sangramiento fue aproximadamente $121 \mathrm{cc}$, excluyendo 2 casos en que fue necesario realizar osteotomía a cielo abierto, En todos los alargamientos femorales bilaterales, se logró los $8 \mathrm{~cm}$ y el alargamiento promedio en esta serie fue de $7,25 \mathrm{~cm}$. Paley, ${ }^{21}$ en una serie inicial de 48 pacientes (65 segmentos) logró alargamiento de $4,41 \mathrm{~cm}$ en promedio $(0,5-6,5)$. En su serie, con 15 casos de Fémur bilateral, el alargamiento promedio fue de 4,63 cm (2,7-6,5 cm). El mismo autor, ${ }^{22}$ publica un estudio con 51 pacientes sometidos a alargamiento bilateral: Se utilizó el clavo Precice P1 (modelo original) en 7 pacientes de Tibia y 22 de Fémur. El clavo P2 (modelo mejorado) fue utilizado en 4 pacientes de Tibia y 25 de Fémur. El alargamiento promedio logrado fue de $5,2 \mathrm{~cm}$ para el grupo $\mathrm{P}$ y $6,0 \mathrm{~cm}$ para el P2.

Los tiempos de consolidación, medidos con el HI fueron de 46,5 días $/ \mathrm{cm}$. Aunque esa cifra es similar a la reportada en diversos estudios ${ }^{7,12,23,24}$ la presente serie incluye tanto alargamiento de Fémur como Tibia, en pacientes de diferentes edades y con diagnósticos de base diferentes, lo que hace poco fiable la comparación.
En los casos bilaterales, 4 de 5 pacientes presentaron retardo de consolidación en uno de los Fémures. Una explicación para ese fenómeno es la falta de adherencia a las indicaciones de limitación de la carga. De acuerdo a las indicaciones del fabricante, para clavos de diámetro $12,5 \mathrm{~mm}$, la carga debiera ser de $34 \mathrm{Kg}$, mientras que para los clavos de 10,7, la carga máxima debiera ser de $22 \mathrm{Kg}$. El uso de IML, permite al paciente rápidamente recuperar la movilidad de sus articulaciones y disminuye en forma considerable el dolor, en comparación con los fijadores externos. Eso les da la falsa impresión de que el hueso está en condiciones de cargar peso, a pesar de las recomendaciones. Paley, ${ }^{21}$ en 15 pacientes con alargamiento bilateral de Fémur reportó 3 fallas del implante en 2 pacientes, debido a carga no autorizada de peso. En su estudio comparativo, ${ }^{22}$ reportó 4 roturas del implante, en 3 pacientes con clavo P1, ocurridos en el día 96,119 y 175 post operatorio. En el grupo de P2, sólo se reportó una rotura del clavo. Todos los casos de falla, se debieron a carga antes de consolidación completa.

En la presente serie, 1 paciente con alargamiento bilateral de Fémur, desarrolló contracturas articulares de cadera y rodilla por no realizar kinesiterapia. Ese paciente, sólo acudió a 3 controles post operatorios, a pesar de haberse contactado en varias oportunidades. A diferencia de los otros pacientes sometidos a la misma intervención, la evaluación psicológica no fue realizada por la psicóloga de nuestro equipo, quien cuenta con una gran experiencia en el examen de esos 

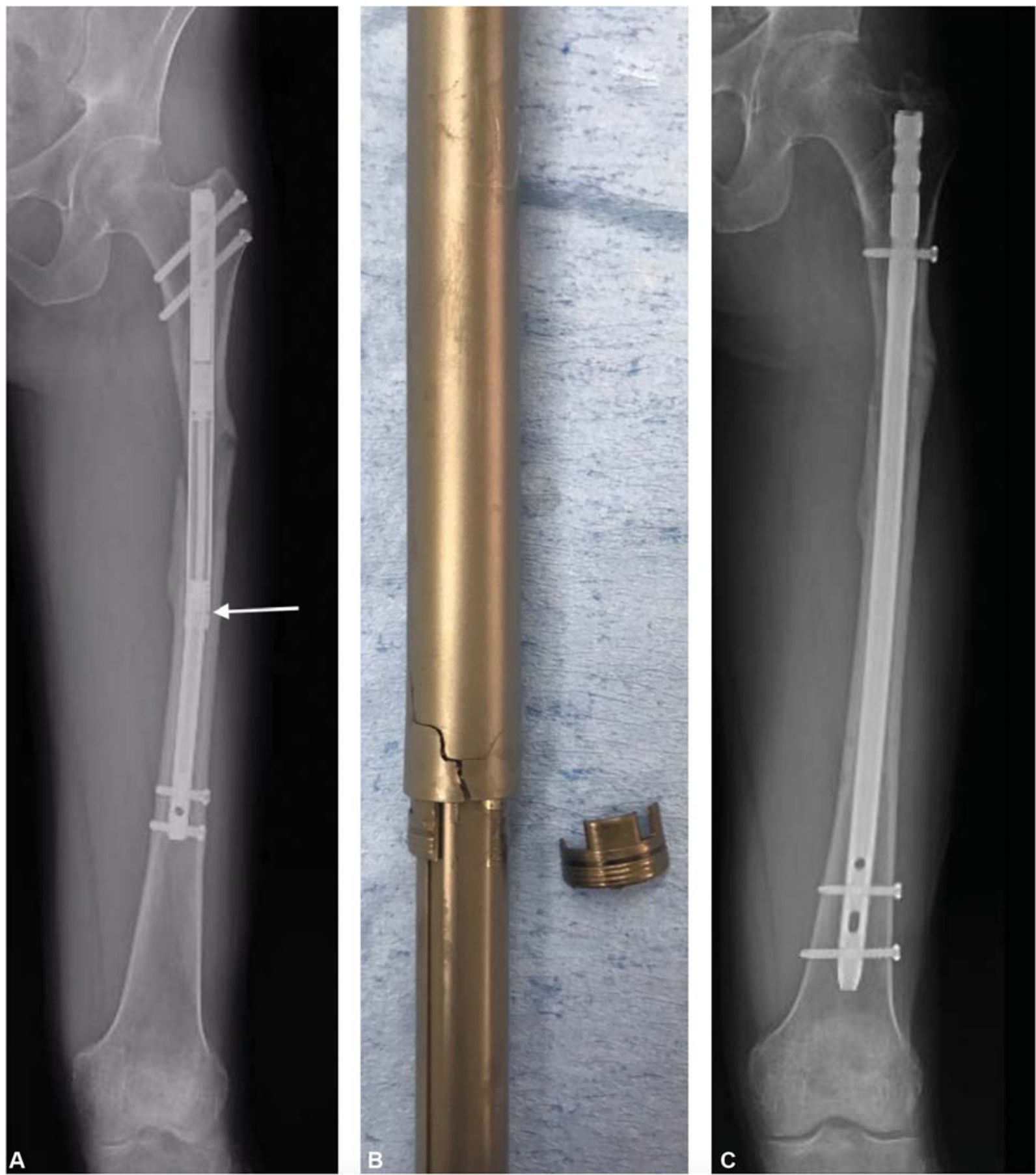

Fig. 3 Deformidad y rotura del implante en Fémur izquierdo. (A): La flecha demuestra la zona de la rotura. (B): Aspecto del clavo al momento del retiro. (C): Se observa el clavo intramedular estándar (R/AFN, DePuy Synthes, Johnson\&Johnson, New Jersey, USA).

pacientes. Eso no hace más que confirmar la importancia de un equipo multidisciplinario, que incluye al cirujano, kinesiólogo, fisiatra y psicólogo ${ }^{24,25}$ que puedan evaluar en conjunto y conversar directamente respecto a cada paciente en particular.

La lesión del N. Peroneo común registrada en 1 paciente, sometido a osteotomía correctora y alargamiento de ambas Tibias, ocurrió durante la perforación para el tornillo de bloqueo más proximal. Si se considera la anatomía del clavo, los bloqueos proximales están dispuestos de manera oblicua, y dependiendo de la profundidad de inserción del clavo, pueden quedar dirigidos directamente hacia el cuello de la Fíbula. De hecho, esa situación es aprovechada por distintos cirujanos, para realizar la Osteodesis Tibio-Fibular proximal, utilizando el tornillo de bloqueo del clavo.

En una serie de 102 fracturas cerradas de Tibia estabilizadas con clavo intramedular, Williams ${ }^{26}$ reporta 19 de lesiones del Nervio Peroneo, no presentes antes de la cirugía, de los cuales 15 se recuperaron completamente. No se explica el mecanismo de la lesión. Por su parte, en trabajos más recientes, $\mathrm{Hems}^{27}$ y Drosos $^{28}$ reportan 1 caso de lesión del Nervio peroneo durante la perforación de uno de los tornillos de bloqueo proximal de dirección oblicua. 
El presente trabajo adolece de significancia estadística por tratarse de una serie de pocos casos, sin embargo, permite sacar algunas lecciones, que permitirán, en el futuro, evitar complicaciones.

Aunque la curva de aprendizaje del alargamiento con IML es mucho menor que con los fijadores externos, aún debe prestarse mucha atención a los detalles.

En la etapa preoperatoria, es fundamental contar con una planificación acuciosa, de manera de elegir el implante ideal para cada paciente, en términos de diámetro, longitud y sitio de entrada. Debe considerarse la existencia de alguna deformidad ósea concomitante, puesto que puede ser corregido de manera aguda y estabilizada con el clavo. Para ello, es de gran ayuda el uso de tornillos de bloqueo, que deben ser instalados estratégicamente, antes de insertar el clavo y sin obstaculizar el paso de este.

Además, de igual importancia, es la evaluación psicológica de aquellos pacientes que quieren someterse a alargamiento de ambas extremidades. Esa evaluación, debiera realizarla un profesional familiarizado con el procedimiento, y que trabaje en estrecho contacto con el cirujano y el equipo e rehabilitación. De esa manera, se pueden unificar criterios, y estandarizar los test a realizar. Durante la cirugía, hay que procurar realizar una osteotomía de baja energía, evitando que se desplacen los fragmentos, puesto de el IML no es canulado, y no se puede utilizar la guía de los clavos tradicionales.

La inserción debe realizarse sin fuerza, y para ello, el canal debe fresarse $2 \mathrm{~mm}$ más ancho que el implante seleccionado.

En la Tibia, se debe ser muy cuidadoso al momento de perforar los tornillos de bloqueo proximal, debido a la disposición oblicua de éstos, con riesgo de lesionar N. Peroneo.

Finalmente, en los casos bilaterales, es de extrema importancia que el paciente comprenda la restricción de la carga hasta que hayan signos categóricos de consolidación, independientemente de los síntomas del paciente.

En suma, los IML ofrecen una ventaja tremenda en términos de comodidad, tolerancia, precisión y control del alargamiento óseo.

Es de esperar que en el futuro cercano, la aparición de nuevos implantes permita una disminución de su valor comercial y de esa manera se logre utilizar con mayor frecuencia en aquellos pacientes que requieren alargamiento óseo.

Conflicto de Intereses

Los autores declaran no tener ningún conflicto de intereses.

\section{Bibliografía}

1 Codivilla A. On the means of lengthening in the lower limbs, the muscles, and tissues which are shortened through deformity. Am J Orthop Surg (Phila Pa) 1905;2:353-369

2 Gubin AV, Borzunov DY, Marchenkova LO, Malkova TA, Smirnova IL. Contribution of G.A. Ilizarov to bone reconstruction: historical achievements and state of the art. Strateg Trauma Limb Reconstr 2016;11(03):145-152

3 Paley D. Problems, obstacles, and complications of limb lengthening by the Ilizarov technique. Clin Orthop Relat Res 1990;(250):81-104
4 Dahl MT, Gulli B, Berg T. Complications of limb lengthening. A learning curve. Clin Orthop Relat Res 1994;(301):10-18

5 Griffith SI, McCarthy JJ, Davidson RS. Comparison of the complication rates between first and second (repeated) lengthening in the same limb segment. J Pediatr Orthop 2006;26(04):534-536

6 Hosny GA. Limb lengthening history, evolution, complications and current concepts. J Orthop Traumatol 2020;21(01):3

7 Calder PR, Laubscher M, Goodier WD. The role of the intramedullary implant in limb lengthening. Injury 2017;48 (Suppl 1):S52-S58

8 Betz A, Baumgart R, Schweiberer L. Erstes voll implantierbares intramedulläres System zur Callusdistraktion-Marknagel mit programmierbarem Antrieb zur Beinverlängerung und Segmentverschiebung. Grundlagen und erste klinische Ergebnisse. [First fully implantable intramedullary system for callus distraction-intramedullary nail with programmable drive for leg lengthening and segment displacement. Principles and initial clinical results] Chirurg 1990;61(08):605-609

9 Paley D. PRECICE intramedullary limb lengthening system. Expert Rev Med Devices 2015;12(03):231-249

10 Fragomen AT, Rozbruch SR. Retrograde magnetic internal lengthening nail for acute femoral deformity correction and limb lengthening. Expert Rev Med Devices 2017;14(10):811-820

11 Iobst CA, Rozbruch SR, Nelson S, Fragomen A. Simultaneous Acute Femoral Deformity Correction and Gradual Limb Lengthening Using a Retrograde Femoral Nail: Technique and Clinical Results. J Am Acad Orthop Surg 2018;26(07):241-250

12 Horn J, Hvid I, Huhnstock S, Breen AB, Steen H. Limb lengthening and deformity correction with externally controlled motorized intramedullary nails: evaluation of 50 consecutive lengthenings. Acta Orthop 2019;90(01):81-87

13 Steiger CN, Lenze U, Krieg AH. A new technique for correction of leg length discrepancies in combination with complex axis deformities of the lower limb using a lengthening nail and a locking plate. J Child Orthop 2018;12(05):515-525

14 Lenze U, Krieg AH. Intramedullary lengthening nails: can we also correct deformities? J Child Orthop 2016;10(06):511-516

15 Muthusamy S, Rozbruch SR, Fragomen AT. The use of blocking screws with internal lengthening nail and reverse rule of thumb for blocking screws in limb lengthening and deformity correction surgery. Strateg Trauma Limb Reconstr 2016;11(03):199-205

16 Hammouda AI, Jauregui JJ, Gesheff MG, Standard SC, Herzenberg JE. Trochanteric Entry for Femoral Lengthening Nails in Children: Is It Safe? J Pediatr Orthop 2017;37(04):258-264

17 Frommer A, Rödl R, Gosheger G, Vogt B. Anwendung motorisierter Verlängerungsmarknägel am wachsenden Skelett : Indikationsspektrum und Limitationen. [Application of motorized intramedullary lengthening nails in skeletally immature patients: Indications and limitations] Unfallchirurg 2018;121(11):860-867

18 Gordon JE, Manske MC, Lewis TR, O'Donnell JC, Schoenecker PL, Keeler KA. Femoral lengthening over a pediatric femoral nail: results and complications. J Pediatr Orthop 2013;33(07):730-736

19 Gordon JE, Swenning TA, Burd TA, Szymanski DA, Schoenecker PL. Proximal femoral radiographic changes after lateral transtrochanteric intramedullary nail placement in children. J Bone Joint Surg Am 2003;85(07):1295-1301

20 Richardson SS, Schairer WW, Fragomen AT, Rozbruch SR. Cost Comparison of Femoral Distraction Osteogenesis With External Lengthening Over a Nail Versus Internal Magnetic Lengthening Nail. J Am Acad Orthop Surg 2019;27(09):e430-e436

21 Paley D, Harris M, Debiparshad K, Prince D. Limb lengthening by implantable limb lengthening devices. Tech Orthop 2014;29(02): 72-85

22 Paley D, Debiparshad K, Balci H, Windisch W, Lichtblau C. Stature Lengthening Using the PRECICE Intramedullary Lengthening Nail. Tech Orthop 2015;30(03):167-182 
23 Calder PR, McKay JE, Timms AJ, et al. Femoral lengthening using the Precice intramedullary limb-lengthening system: outcome comparison following antegrade and retrograde nails. Bone Joint J 2019;101-B(09):1168-1176

24 Wagner P, Burghardt RD, Green SA, Specht SC, Standard SC, Herzenberg JE. PRECICE ${ }^{\circledR}$ magnetically-driven, telescopic, intramedullary lengthening nail: pre-clinical testing and first 30 patients. SICOT J 2017;3:19

25 Paley D, Windisch W. Cosmetic limb lengthening for stature: Ethics, Methods and Results. Perth, Australia: Australian Orthopaedic Association; 2005
26 Williams J, Gibbons M, Trundle H, Murray D, Worlock P. Complications of nailing in closed tibial fractures. J Orthop Trauma 1995;9(06):476-481

27 Hems TE, Jones BG. Peroneal nerve damage associated with the proximal locking screws of the AIM tibial nail. Injury 2005;36 (05):651-654, discussion 655

28 Drosos GI, Stavropoulos NI, Kazakos KI. Peroneal nerve damage by oblique proximal locking screw in tibial fracture nailing: a new emerging complication? Arch Orthop Trauma Surg 2007;127(06): 449-451 